\section{Avaliação do desempenho dos serviços de atenção primária à saúde no controle da tuberculose em metrópole do Sudeste do Brasil}

\section{Performance assessment of primary healthcare services in tuberculosis control in a city in Southeast Brazil}

\section{Evaluación del desempeño de los servicios de atención primaria en salud para el control de la tuberculosis en metrópolis del sudeste de Brasil}

\section{Resumo}

A Organização Mundial da Saúde destaca a importância da organização e do desempenho dos serviços de saúde nas ações de controle da tuberculose. $O$ objetivo deste estudo foi avaliar o desempenho dos serviços de atenção primaria à saúde em Belo Horizonte, Minas Gerais, Brasil, em relação às ações de controle da tuberculose nos eixos Estrutura e Processo, antes e após a utilização do instrumento validado denominado Estratificação por Grau de Risco Clínico e de Abandono do Tratamento da Tuberculose (ERTB). Estudo descritivo e prospectivo, no qual foram realizadas duas entrevistas (455 profissionais), tendo a segunda ocorrido após a ERTB. A classificação do desempenho seguiu os valores: $\leq 49,9 \%$, críticos; entre 50\% e 79,9\%, insatisfatórios; $e, \geq 80 \%$, satisfatórios. $\mathrm{Na}$ avaliação comparativa, foi utilizado o teste estatístico de McNemar, com $p<0,05$. Após a estratificação de risco, a maior parte das variáveis de cada eixo melhorou significativamente. Nós concluímos que, por meio de um questionário padronizado, foi possível identificar o desempenho satisfatório dos serviços de atenção primária à saúde em Belo Horizonte em relação às ações de controle da tuberculose, na maioria das variáveis avaliadas nos eixos Estrutura e Processo.

Tuberculose; Serviços de Saúde; Atenção Primária à Saúde
Juliana Veiga Costa Rabelo 1 Pedro Daibert de Navarro ${ }^{1}$ Wânia da Silva Carvalho 2 Isabela Neves de Almeida 3 Camila Stefânie Fonseca Oliveira 4 João Paulo Amaral Haddad 4 Silvana Spindola de Miranda 3

doi: 10.1590/0102-311X00112020

\author{
rrespondência \\ S. S. Miranda \\ Faculdade de Medicina, Universidade Federal de Minas Gerais. \\ Av. Alfredo Balena 190, Belo Horizonte, MG 30130-100, Brasil. \\ silvanaspindola@gmail.com
1 Secretaria Municipal de Saúde de Belo Horizonte, Belo Horizonte, Brasil.
2 Faculdade de Farmácia, Universidade Federal de Minas Gerais, Belo Horizonte, Brasil.
3 Faculdade de Medicina, Universidade Federal de Minas Gerais, Belo Horizonte, Brasil.
4 Escola de Veterinária, Universidade Federal de Minas Gerais, Belo Horizonte, Brasil.




\section{Introdução}

A tuberculose (TB), causada pelo Mycobacterium tuberculosis, é uma doença infecciosa existente há milhares de anos e ainda considerada um grave problema de saúde global 1. No ano de 1993, a Organização Mundial da Saúde (OMS), declarou a TB como uma das emergências mundiais juntamente com a malária e a aids 2,3; em 1996, a TB passa a ser prioridade sanitária devido ao grande desafio do quadro epidemiológico 4. Em 2019, manteve-se entre as 10 principais causas de óbito por doenças infecciosas causadas por agente único no mundo 1.

A OMS destaca ainda a importância da organização e do desempenho dos serviços de saúde nas ações de controle da TB, afirmando que o problema não está somente na detecção e tratamento da doença, mas também na forma como os serviços se organizam para detectar e tratar esses casos 5 .

A atenção primária à saúde (APS) tem por objetivo o cumprimento de três funções essenciais: resolubilidade (solucionar mais de $85 \%$ dos problemas da população), comunicação (ordenar os fluxos e contrafluxos de pessoas, produtos e informações entre os diferentes níveis de atenção) e responsabilização pelos microterritórios sanitários ${ }^{6}$. Assim, a porta de entrada aos serviços de saúde deve ser preferencialmente pela APS, e, quando se fala da TB, esses serviços devem desempenhar o papel de coordenadoria do cuidado, desenvolvimento de ações de promoção, prevenção e controle da doença 7,8,9.

Na primeira década do século XXI, o Brasil vivenciou mudanças na organização dos serviços de saúde, e a APS recebeu enfoque maior com a introdução de programas estratégicos para a mudança do modelo assistencial no Sistema Único de Saúde (SUS) 6,9. Apesar dessas mudanças, os desfechos cura e abandono de tratamento da TB permanecem aquém do preconizado ( $85 \%$ e $5 \%$ respectivamente), principalmente em Belo Horizonte, Minas Gerais, uma das grandes capitais do Brasil, pioneira na implantação da Estratégia de Saúde da Família (ESF) 3,10. Em 2018, 73,5\% e 66,7\% foram curados, enquanto 11,8\% e 13,2\% abandonaram o tratamento, no Brasil e em Belo Horizonte respectivamente 11.

Nesse contexto, a avaliação do desempenho dos serviços é necessária para qualificar o modelo vigente e direcionar ações para melhoria dos serviços prestados aos pacientes com a doença.

Diante do exposto, o objetivo desse estudo foi avaliar o desempenho dos serviços de APS em Belo Horizonte em relação às ações de controle da TB nos eixos Estrutura e Processo, antes e após a utilização do instrumento validado denominado Estratificação por Grau de Risco Clínico e de Abandono do Tratamento da Tuberculose (ERTB) 10,12.

\section{Métodos}

Estudo descritivo, prospectivo, realizado em Belo Horizonte, parte do Projeto de Ações Contingenciais para o Enfrentamento da Tuberculose em Belo Horizonte financiado pelo Ministério da Saúde, por meio da Portaria no 3.110, de 17 de dezembro de 2013 13. Foram contratados 14 profissionais de nível superior para execução do mesmo.

A APS em Belo Horizonte é composta por 588 equipes de saúde da família (EqSF) distribuídas em 152 centros de saúde, correspondendo a uma cobertura de $88 \%$. Apresenta, ainda, 100\% de cobertura de Núcleo de Apoio à Estratégia Saúde da Família (NASF) composto, dentre outras categorias, por farmacêutico, assistente social e psicólogo. O atendimento aos pacientes com TB é descentralizado para toda a APS, que funciona em horário comercial ( $7 \mathrm{~h}$ às $18 \mathrm{~h}$ ), sendo realizado por toda a equipe dos centros de saúde 10 .

Em setembro de 2016, os pacientes com TB de metade dos centros de saúde foram estratificados pelo grau de risco clínico e de abandono do tratamento da doença, por meio de um instrumento denominado ERTB 10,12, elaborado por um grupo de técnicos da Secretaria Municipal de Saúde de Belo Horizonte. $\mathrm{O}$ instrumento é estruturado em duas partes: a primeira classifica o risco de abandono do tratamento (baixo ou alto) e a segunda, o risco clínico (baixo, médio, alto e muito alto), de acordo com a forma da doença, presença de comorbidades, resistência bacteriana e intercorrências clínicas (acompanhamento pela equipe do NASF e, quando necessário, pela equipe da saúde mental, assistência social, entre outros), além de informação sobre a doença e tratamento (encaminhamento para a atenção primária, secundária ou terciária, segundo preconizado pelo Ministério da Saúde) 3,10,12. 
Os dados foram coletados em duas etapas: julho a agosto de 2016 (primeira entrevista), quando ainda não havia sido aplicado o instrumento de ERTB; julho a setembro de 2017 (segunda entrevista), um ano após a utilização do mesmo instrumento em metade dos centros de saúde.

Para evitar viés de seleção na amostra, todas as unidades de APS foram divididas em dois grupos (um grupo foi classificado por ERTB e outro não), observando a organização administrativa da rede de saúde, a presença das áreas de cluster para a TB no território e o Índice de Vulnerabilidade em Saúde 14. Esse índice é um indicador sintético que agrega vários indicadores sociais, econômicos e ambientais, mais ou menos complexos, para analisar as características de grupos populacionais vivendo em determinadas áreas geográficas. Para a definição da existência de possíveis clusters, foi necessário georreferenciar os casos de 2012 a 2015 utilizando o endereço e a aplicação presentes no seguinte site da Internet: https://www.mapdevelopers.com/batch_geocode_tool.php. Outro aplicativo utilizado para avaliar a presença de cluster foi o SaTScan (http:\\www.satscan.org), utilizando-se definições da configuração à procura por "altas taxas", "puramente espacial" com o modelo de Poisson discreto.

Optou-se por entrevistar apenas os profissionais de nível superior, médico ou enfermeiro, de cada uma das $588 \mathrm{EqSF}$, por serem estes os profissionais que faziam as ações de controle da TB nos 152 centros de saúde. Foram incluídos os profissionais que responderam ao questionário nas duas etapas. Para as entrevistas, utilizou-se um questionário estruturado, validado nacionalmente 15 , composto por 44 questões, que tiveram algumas adaptações autorizadas pelos autores originais para adequar-se à realidade do município.

As variáveis selecionadas para responder aos objetivos do estudo correspondem aos eixos Estrutura (Recursos Humanos, Recursos Físicos e Organização do Serviço) e Processo (Atenção Proporcionada) (Quadro 1). Foi realizada uma única modificação no instrumento original em relação ao Teste Rápido Molecular, que não existia no instrumento original (Quadro 1). O software Stata versão 14.0 (https://www.stata.com) foi utilizado para análise dos dados.

A etapa de análise e classificação do desempenho dos serviços de saúde da APS segundo os eixos Estrutura e Processo, coletados a partir de dados primários, baseou-se nos estudos de Samico et al. $16 \mathrm{e}$ Felisberto et al. 17 , tendo sido utilizada por Silva 18 na avaliação das ações de controle da TB. Os indicadores foram analisados de acordo com a frequência de cada variável estudada (proporção de respostas).

A classificação do desempenho dos serviços de saúde seguiu valores obtidos dos indicadores: valores menores de 49,9\% foram avaliados como críticos, entre 50\% e 79,9\%, insatisfatórios e maiores ou iguais a $80 \%$, satisfatórios (Quadro 2).

Para a avaliação comparativa após a ERTB e entre os grupos que foram e que não foram classificados por ERTB, foi utilizado o teste estatístico de comparação McNemar, sendo considerado significativo $\mathrm{p}<0,05$ e a odds ratio (OR). O teste foi realizado em duas etapas, a primeira considerando antes e após a ERTB e a segunda considerando os grupos (os que foram e os que não foram classificados por ERTB) (Figura 1).

Este estudo foi aprovado pelo Comitê de Ética em Pesquisa da Universidade Federal de Minas Gerais: Estratificação por Grau de Risco Clínico e de Abandono do Tratamento da Tuberculose em Pacientes Acompanhados nos Serviços de Atenção Primária à Saúde de Belo Horizonte - CAAE: 43320015.4.0000.5149.

\section{Resultados}

Foram incluídos 455 médicos e enfermeiros que participaram das duas entrevistas.

O desempenho dos serviços da APS para os indicadores dos eixos Estrutura e Processo na atenção à TB pode ser observado na Tabela 1 .

No eixo Estrutura - Recursos Humanos, nota-se que, na primeira entrevista, a variável "envolvimento de outros profissionais com o atendimento ao doente de TB" foi classificada como insatisfatória $(56,5 \%)$, mas apresenta melhora significativa na segunda entrevista $(99 \%)$.

No eixo Estrutura - Recursos Físicos, a variável "disponibilidade de pote para exame de escarro" teve seu desempenho insatisfatório na segunda entrevista em relação à primeira $(95 \%$ e 72,8\%, respectivamente). Essa diferença não foi significativa (Tabelas 1 e 2).

No eixo Estrutura - Organização do Serviço, as variáveis "atendimento fora do horário comercial" e "articulação dos serviços de APS com outros níveis de atenção" apresentaram, na segunda entrevista, 
Quadro 1

Variáveis de desempenho dos serviços da atenção primária à saúde (APS) no tratamento da tuberculose (TB), segundo os eixos avaliativos dos serviços de saúde.

\begin{tabular}{|c|c|}
\hline \multicolumn{2}{|r|}{ Estrutura } \\
\hline Eixo & Variáveis \\
\hline \multirow[t]{8}{*}{ Recursos Humanos } & Equipe completa de profissionais de saúde envolvidos com o atendimento aos doentes de TB \\
\hline & Envolvimento de outros profissionais com o atendimento aos doentes de TB \\
\hline & Envolvimento de equipe completa de outros profissionais com o atendimento aos doentes de TB \\
\hline & Capacitação em TB para pelo menos um profissional da equipe de atendimento aos doentes nos serviços de saúde \\
\hline & $\begin{array}{c}\text { Capacitação para atendimento em TB da equipe mínima de profissionais de saúde (enfermeiro, médico, técnico de } \\
\text { enfermagem, agentes comunitários de saúde }\end{array}$ \\
\hline & Oferta de capacitação em TB pelo PCT municipal \\
\hline & Oferta de capacitação em TB pelos serviços de APS \\
\hline & Profissional de saúde responsável pelo tratamento diretamente observado \\
\hline \multirow[t]{15}{*}{ Recursos Físicos } & Disponibilidade de salas para atendimento médico e de enfermagem \\
\hline & Disponibilidade de cestas básicas \\
\hline & Disponibilidade de vale-transporte \\
\hline & Acesso à ficha de notificação \\
\hline & Acesso aos prontuários dos doentes de TB \\
\hline & Acesso à ficha de acompanhamento mensal \\
\hline & Acesso à ficha diária de tratamento diretamente observado \\
\hline & Acesso ao livro registro de sintomáticos respiratórios \\
\hline & Acesso ao livro de acompanhamento de casos \\
\hline & Disponibilidade de pote para exame de escarro \\
\hline & Disponibilidade de pedido de baciloscopia de escarro/TRM-TB \\
\hline & Disponibilidade de pedido de cultura de escarro \\
\hline & Disponibilidade de pedido de exame de HIV \\
\hline & Disponibilidade de ficha de referência e contrarreferência \\
\hline & Disponibilidade de medicamentos para o tratamento da TB \\
\hline \multirow[t]{9}{*}{ Organização do Serviço } & Atendimento fora do horário comercial \\
\hline & Discussões dos casos de TB entre profissionais de saúde responsáveis pelo atendimento \\
\hline & Acesso à consulta em prazo de 24 horas \\
\hline & Orientação quanto à busca por atendimento fora da data agendada caso necessite \\
\hline & Articulação dos todos os serviços de APS com outros níveis de atenção \\
\hline & Articulação de pelo menos um dos serviços de APS com outros níveis de atenção \\
\hline & Atendimento laboratorial da demanda aos serviços de APS atende a demanda \\
\hline & Acesso aos resultados do exame de baciloscopia de escarro/TRM-TB em sete dias \\
\hline & Existência de sistema informatizado contendo informações sobre doente de TB \\
\hline \multicolumn{2}{|r|}{ Processo } \\
\hline Eixo & Variáveis \\
\hline \multirow[t]{12}{*}{ Atenção Proporcionada } & Orientação ao doente de TB quanto à doença \\
\hline & Discussão conjunta com doente de TB quanto à forma de realização do seu acompanhamento \\
\hline & Oferta de tratamento diretamente observado no domicílio \\
\hline & Oferta de tratamento diretamente observado no serviço de saúde \\
\hline & Oferta de tratamento diretamente observado no trabalho do doente de TB \\
\hline & Realização mínima de baciloscopia mensal de escarro para controle do tratamento \\
\hline & Realização de consulta médica mensal e tratamento diretamente observado no mesmo serviço de saúde \\
\hline & Auxílio no agendamento de consultas/exames quando doente de TB é encaminhado a outros serviços \\
\hline & Fornecimento de informações escritas aos doentes encaminhados a outros serviços de saúde \\
\hline & Existência de contrarreferência das informações \\
\hline & Busca ao doente de TB quando não comparece à consulta \\
\hline & Busca ao doente de TB quando não realiza tratamento diretamente observado \\
\hline
\end{tabular}

PCT: Programa de Controle da Tuberculose; TRM: teste rápido molecular.

Fonte: Questionário dos serviços de APS no tratamento da tuberculose, 2014; adaptado pelo autor. 


\section{Quadro 2}

Critérios para interpretação dos resultados obtidos para os eixos avaliativos e variáveis.

\begin{tabular}{|c|c|c|}
\hline Eixos avaliativos & Ponto de corte (\%) & Interpretação \\
\hline Estrutura * e Processo ** & $\leq 49,9$ & Crítico \\
\cline { 2 - 3 } & 50,0 a 79,9 & Insatisfatório \\
\cline { 2 - 3 } & $\geq 80,0$ & Satisfatório \\
\hline
\end{tabular}

Fonte: Samico et al. 16 e Felisberto et al. 17; adaptado pelos autores.

* Estrutura: Recursos Humanos, Recursos Físicos e Organização do Serviço;

** Processo: Atenção Proporcionada.

\section{Figura 1}

Etapas da realização da Estratificação por Grau de Risco Clínico e de Abandono do Tratamento da Tuberculose (ERTB). Belo Horizonte, Minas Gerais, Brasil.

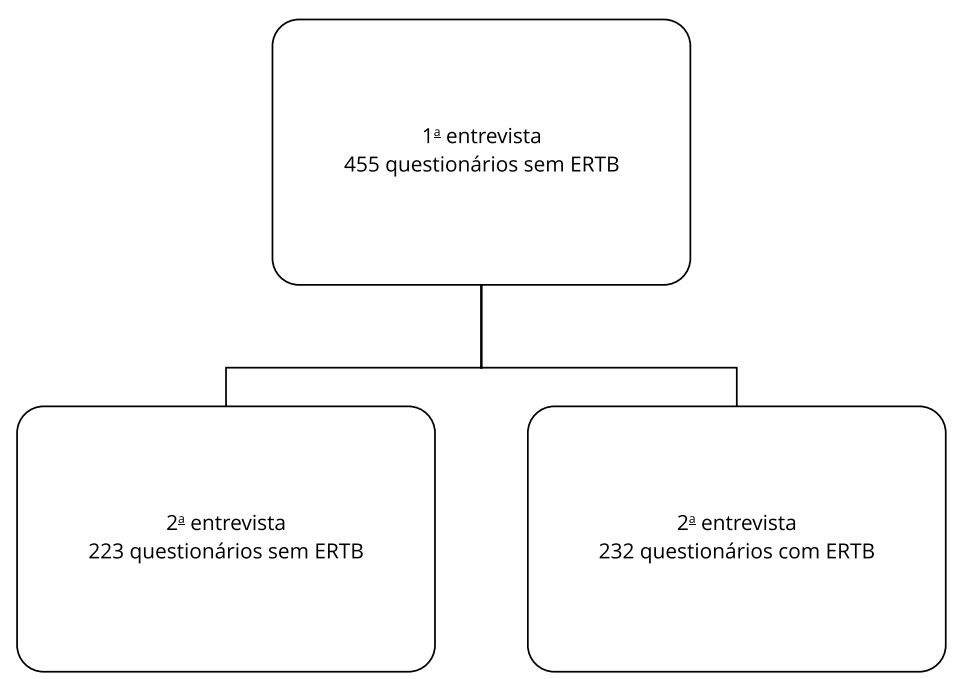

melhora significativa no desempenho passando a ser satisfatórias (75\% e 80,9\%; 74,3\% e 80,2\%; respectivamente) (Tabelas 1 e 2). A variável "Acesso aos resultados do exame de baciloscopia de escarro/ teste rápido molecular em sete dias”, apresentou, na segunda entrevista desempenho satisfatório em relação à primeira (75,8\% e 90,1\%), porém não foi significativo (Tabelas 1 e 2).

Em relação ao eixo Processo - Atenção Proporcionada, não houve alterações na primeira entrevista quando comparada com a segunda (Tabela 1).

As classificações nos eixos Estrutura - Recursos Humanos, Recursos Físicos e Atenção Proporcionada, apresentaram classificação crítica na primeira e segunda entrevistas. Não foi observada classificação crítica em relação à Estrutura - Organização de Serviço (Tabela 1).

Das 44 variáveis de desempenho estudadas, 16 apresentaram diferença estatística após a ERTB (Tabela 2).

É possível observar que nove variáveis apresentaram diferença estaticamente significativa quando comparamos as associações entre as variáveis de avaliação de desempenho nos grupos que realizaram 
Tabela 1

Distribuição proporcional e classificação das variáveis, por eixo, dos serviços de atenção primária à saúde (APS) para o tratamento da tuberculose, segundo os profissionais de saúde. Belo Horizonte, Minas Gerais, Brasil, 2016 e 2017.

\begin{tabular}{|c|c|c|c|c|}
\hline \multirow[t]{2}{*}{ Eixo/Variáveis } & \multicolumn{2}{|r|}{ 1a etapa } & \multicolumn{2}{|r|}{ 2a etapa } \\
\hline & $\operatorname{Sim}(\%)$ & Classificação & $\operatorname{Sim}(\%)$ & Classificação \\
\hline \multicolumn{5}{|l|}{ Estrutura - Recursos Humanos } \\
\hline $\begin{array}{l}\text { Equipe completa de profissionais de saúde envolvidos com o atendimento aos } \\
\text { doentes de TB }\end{array}$ & 89,9 & Satisfatório & 92,8 & Satisfatório \\
\hline Envolvimento de outros profissionais com o atendimento aos doentes de TB & 56,5 & Insatisfatório * & 99,0 & Satisfatório * \\
\hline $\begin{array}{l}\text { Envolvimento de equipe completa de outros profissionais com o atendimento aos } \\
\text { doentes de TB }\end{array}$ & 14,3 & Crítico ** & 43,1 & Crítico ** \\
\hline $\begin{array}{l}\text { Capacitação em TB para pelo menos } 1 \text { profissional da equipe de atendimento aos } \\
\text { doentes nos serviços de saúde }\end{array}$ & 67,3 & Insatisfatório ** & 60,0 & Insatisfatório ** \\
\hline $\begin{array}{l}\text { Capacitação para atendimento em TB à equipe mínima de profissionais de saúde } \\
\text { (enfermeiro, médico, enfermeiro nível médio, ACS) }\end{array}$ & 7,0 & Crítico ** & 4,4 & Crítico ** \\
\hline Oferta de capacitação em TB pelo PCT municipal & 30,3 & Crítico ** & 20,7 & Crítico ** \\
\hline Oferta de capacitação em TB pelos serviços de atenção básica & 18,5 & Crítico ** & 20,4 & Crítico ** \\
\hline Profissional de Saúde responsável pelo tratamento diretamente observado & 86,6 & Satisfatório & 93,9 & Satisfatório \\
\hline \multicolumn{5}{|l|}{ Estrutura - Recursos Físicos } \\
\hline Disponibilidade de salas para atendimento médico e de enfermagem & 91,0 & Satisfatório & 84,2 & Satisfatório \\
\hline Disponibilidade de cestas básicas & 7,5 & Crítico ** & 3,1 & Crí́tico ** \\
\hline Disponibilidade de vale-transporte & 4,8 & Crítico ** & 2,2 & Crítico** \\
\hline Acesso à ficha de notificação & 99,3 & Satisfatório & 99,0 & Satisfatório \\
\hline Acesso aos prontuários dos doentes de TB & 100,0 & Satisfatório & 100,0 & Satisfatório \\
\hline Acesso à ficha de acompanhamento mensal & 99,6 & Satisfatório & 98,9 & Satisfatório \\
\hline Acesso à ficha diária de tratamento diretamente observado & 96,5 & Satisfatório & 99,1 & Satisfatório \\
\hline Acesso ao livro de registro de sintomáticos respiratórios & 99,8 & Satisfatório & 99,8 & Satisfatório \\
\hline Acesso ao livro de acompanhamento de casos & 96,9 & Satisfatório & 99,8 & Satisfatório \\
\hline Disponibilidade de pote para exame de escarro & 95,0 & Satisfatório *** & 72,8 & Insatisfatório *** \\
\hline Disponibilidade de pedido de baciloscopia de escarro/teste rápido molecular & 98,5 & Satisfatório & 100,0 & Satisfatório \\
\hline Disponibilidade de pedido de cultura de escarro & 98,0 & Satisfatório & 99,8 & Satisfatório \\
\hline Disponibilidade de pedido de exame de HIV & 100,0 & Satisfatório & 100,0 & Satisfatório \\
\hline Disponibilidade de ficha de referência e contrarreferência & 97,8 & Satisfatório & 100,0 & Satisfatório \\
\hline Disponibilidade de medicamentos para o tratamento da TB & 96,7 & Satisfatório & 99,6 & Satisfatório \\
\hline \multicolumn{5}{|l|}{ Estrutura - Organização do Serviço } \\
\hline Atendimento fora do horário comercial & 75,0 & Insatisfatório * & 80,9 & Satisfatório * \\
\hline $\begin{array}{l}\text { Discussões dos casos de TB entre profissionais de saúde responsáveis pelo } \\
\text { atendimento }\end{array}$ & 97,4 & Satisfatório & 97,6 & Satisfatório \\
\hline Acesso a consultas em prazo de 24 horas & 99,3 & Satisfatório & 99,8 & Satisfatório \\
\hline $\begin{array}{l}\text { Orientação quanto à busca por atendimento fora da data agendada caso } \\
\text { necessite }\end{array}$ & 98,9 & Satisfatório & 100,0 & Satisfatório \\
\hline Articulação dos todos os serviços de APS com outros níveis de atenção & 74,3 & Insatisfatório * & 80,2 & Satisfatório * \\
\hline Articulação de pelo menos um dos serviços de APS com outros níveis de atenção & 96,0 & Satisfatório & 98,7 & Satisfatório \\
\hline Atendimento laboratorial da demanda aos serviços de APS atende a demanda & 94,7 & Satisfatório & 98,2 & Satisfatório \\
\hline Acesso aos resultados do exame de baciloscopia de escarro/TRM-TB em sete dias & 75,8 & Insatisfatório * & 90,1 & Satisfatório * \\
\hline Existência de sistema informatizado contendo informações sobre doente de TB & 96,9 & Satisfatório & 98,9 & Satisfatório \\
\hline
\end{tabular}

(continua) 
Tabela 1 (continuação)

\begin{tabular}{|c|c|c|c|c|}
\hline \multirow[t]{2}{*}{ Eixo/Variáveis } & \multicolumn{2}{|c|}{ 1ạ etapa } & \multicolumn{2}{|r|}{ 2a etapa } \\
\hline & $\operatorname{Sim}(\%)$ & Classificação & $\operatorname{Sim}(\%)$ & Classificação \\
\hline \multicolumn{5}{|l|}{ Processo - Atenção Proporcionada } \\
\hline Orientação ao doente de TB quanto à doença & 98,5 & Satisfatório & 100,0 & Satisfatório \\
\hline $\begin{array}{l}\text { Discussão conjunta com doente de TB quanto à forma de realização do seu } \\
\text { acompanhamento }\end{array}$ & 89,0 & Satisfatório & 96,0 & Satisfatório \\
\hline Oferta de tratamento diretamente observado no domicílio & 85,1 & Satisfatório & 89,9 & Satisfatório \\
\hline Oferta de tratamento diretamente observado no Serviço de Saúde & 66,4 & Insatisfatório ** & 75,2 & Insatisfatório ** \\
\hline Oferta de tratamento diretamente observado no trabalho do doente de TB & 9,0 & Crítico ** & 6,4 & Crítico ** \\
\hline $\begin{array}{l}\text { Realização mínima de baciloscopia mensal de escarro para controle do } \\
\text { tratamento }\end{array}$ & 64,8 & Insatisfatório ** & 75,2 & Insatisfatório ** \\
\hline $\begin{array}{l}\text { Realização de consulta médica mensal e tratamento diretamente observado no } \\
\text { mesmo serviço de saúde }\end{array}$ & 95,4 & Satisfatório & 99,3 & Satisfatório \\
\hline $\begin{array}{l}\text { Auxílio no agendamento de consultas/exames quando doente de TB é } \\
\text { encaminhado a outros serviços }\end{array}$ & 96,7 & Satisfatório & 98,7 & Satisfatório \\
\hline $\begin{array}{l}\text { Fornecimento de informações escritas aos doentes encaminhados a outros } \\
\text { serviços de saúde }\end{array}$ & 98,2 & Satisfatório & 100,0 & Satisfatório \\
\hline Existência de contrarreferência das informações & 40,7 & Crítico ** & 48,1 & Crítico ** \\
\hline Busca ao doente de TB quando não comparece à consulta & 99,6 & Satisfatório & 100,0 & Satisfatório \\
\hline Busca ao doente de TB quando não realiza tratamento diretamente observado & 95,0 & Satisfatório & 96,5 & Satisfatório \\
\hline
\end{tabular}

ACS: agente comunitário em saúde; PCT: Programa de Controle da Tuberculose; TB: tuberculose; TRM/TB: teste rápido molecular.

* Melhora no desempenho;

** Desempenho se manteve;

*** Piora no desempenho;

a estratificação (Tabela 2). São elas: "capacitação em TB para pelo menos um profissional da equipe de atendimento aos doentes nos serviços de saúde" e "capacitação para atendimento em TB à equipe mínima de profissionais de saúde (enfermeiro, médico, técnico em enfermagem, agente comunitário em saúde)", apresentando razão de chance de ter desempenho melhor avaliado quando há a ERTB, da ordem de 50\%; "oferta de capacitação em TB pelos serviços de atenção básica”, com 1,7 vez mais chance de ter seu desempenho mais bem avaliado; "disponibilidade de cestas básicas e disponibilidade de vale-transporte", em que a chance é de 0,1 vez ser mais bem avaliado; "atendimento fora do horário comercial”, 2,2 vezes mais chances; "articulação dos serviços de APS com outros níveis de atenção", com uma chance de ter seu desempenho mais bem avaliado em 1,9 vez; "oferta de tratamento diretamente observado no trabalho do doente de TB", duas vezes mais chances; por fim, "existência de contrarreferência das informações” com 1,8 mais chance de ter seu desempenho mais bem avaliado (Tabela 2).

\section{Discussão}

Diversas iniciativas voltadas para a avaliação em saúde no Brasil vêm sendo desenvolvidas com objetivo de qualificar os serviços prestados à população, porém ainda são escassos os estudos sobre o desempenho dos serviços de saúde em relação às ações de controle da TB 19,20,21. Nesse contexto, este trabalho demonstrou que é possível avaliar o desempenho dos serviços de APS, na perspectiva dos profissionais médicos e enfermeiros, utilizando um questionário padronizado.

Observamos que, após a ERTB, a maior parte das variáveis de cada eixo melhorou significativamente, mesmo aquelas que já apresentavam o desempenho satisfatório. Em relação ao envolvimento de outros profissionais, principalmente o farmacêutico do NASF no eixo Estrutura - Recursos Humanos, quando esse foi inserido ativamente no atendimento, houve melhora no desempenho. Os pro- 
Tabela 2

Comparação das associações entre as variáveis de avaliação de desempenho antes e após a estratificação de risco descrita em termos de suas odds ratio (OR), intervalos de $95 \%$ de confiança (IC95\%) e valor de $\mathrm{p}$.

\begin{tabular}{|c|c|c|c|c|}
\hline Eixo/Variáveis & Estratificação & OR & IC95\% & Valor de $p$ \\
\hline \multicolumn{5}{|l|}{ Recursos Humanos } \\
\hline \multirow[t]{2}{*}{ Envolvimento de outros profissionais com o atendimento aos doentes de TB } & Não & 4,4 & $1,625-14,873$ & 0,001 \\
\hline & Sim & 10,5 & $2,566-92,370$ & 0,000 \\
\hline Envolvimento de equipe completa de outros profissionais com o atendimento aos & Não & 3,7 & $2,308-6,140$ & 0,000 \\
\hline doentes de TB & Sim & 3,6 & $2,208-6,179$ & 0,000 \\
\hline Capacitação em TB para pelo menos um profissional da equipe de atendimento & Não & 0,8 & $0,485-1,237$ & 0,263 \\
\hline aos doentes nos serviços de saúde & $\operatorname{Sim}$ & 0,5 & $0,335-0,876$ & 0,008 * \\
\hline Capacitação para atendimento em TB à equipe mínima de profissionais de saúde & Não & 0,7 & $0,426-1,072$ & 0,080 \\
\hline (enfermeiro, médico, técnico em enfermagem, ACS) & Sim & 0,5 & $0,297-0,791$ & 0,002 * \\
\hline \multirow[t]{2}{*}{ Oferta de capacitação em TB pelo PCT municipal } & Não & 0,5 & $0,323-0,940$ & 0,020 \\
\hline & Sim & 0,5 & $0,287-0,813$ & 0,003 \\
\hline \multirow[t]{2}{*}{ Oferta de capacitação em TB pelos serviços de atenção básica } & Não & 0,8 & $0,461-1,472$ & 0,492 \\
\hline & $\operatorname{Sim}$ & 1,7 & $0,968-3,363$ & $0,047 *$ \\
\hline \multicolumn{5}{|l|}{ Recursos Físicos } \\
\hline \multirow[t]{2}{*}{ Disponibilidade de cestas básicas } & Não & 1,0 & $0,025-0,431$ & 1,000 \\
\hline & Sim & 0,1 & $0,351-2,843$ & $0,000 *$ \\
\hline \multirow[t]{2}{*}{ Disponibilidade de vale-transporte } & Não & 0,5 & $0,025-0,431$ & 0,248 \\
\hline & Sim & 0,1 & $0,110-1,866$ & $0,000 *$ \\
\hline \multirow[t]{2}{*}{ Disponibilidade de pote para exame de escarro } & Não & 0,1 & $0,061-0,319$ & 0,000 \\
\hline & $\operatorname{Sim}$ & 0,05 & $0,010-0,156$ & 0,000 \\
\hline \multicolumn{5}{|l|}{ Organização do Serviço } \\
\hline \multirow[t]{2}{*}{ Atendimento fora do horário comercial } & Não & 1,5 & $0,827-2,880$ & 0,148 \\
\hline & Sim & 2,2 & $1,143-4,504$ & $0,011 *$ \\
\hline \multirow[t]{2}{*}{ Articulação dos serviços de APS com outros níveis de atenção } & Não & 1,2 & $0,744-2,009$ & 0,406 \\
\hline & Sim & 1,9 & $1,127-3,477$ & $0,011 *$ \\
\hline \multirow[t]{2}{*}{ Acesso aos resultados do exame de baciloscopia de escarro/TRM-TB em sete dias } & Não & 4,0 & $2,147-8,007$ & 0,000 \\
\hline & Sim & 3,6 & $1,750-8,133$ & 0,000 \\
\hline \multicolumn{5}{|l|}{ Atenção Proporcionada } \\
\hline \multirow[t]{2}{*}{ Oferta de tratamento diretamente observado no Serviço de Saúde } & Não & 1,8 & $1,044-3,112$ & 0,024 \\
\hline & $\operatorname{Sim}$ & 2,6 & $1,353-5,161$ & 0,001 \\
\hline \multirow[t]{2}{*}{ Oferta de tratamento diretamente observado no trabalho do doente de TB } & Não & 2,0 & $0,807-5,397$ & 0,102 \\
\hline & Sim & 0,2 & $0,095-0,610$ & $0,000 *$ \\
\hline \multirow[t]{2}{*}{ Realização mínima de baciloscopia mensal de escarro para controle do tratamento } & Não & 2,1 & $1,219-3,709$ & 0,004 \\
\hline & Sim & 2,5 & $0,807-5,397$ & 0,001 \\
\hline \multirow[t]{2}{*}{ Existência de contrarreferência das informações } & Não & 1,2 & $1,164-3,032$ & 0,345 \\
\hline & Sim & 1,8 & $0,790-1,890$ & 0,006 * \\
\hline
\end{tabular}

ACS: agente comunitário em saúde; APS: atenção primária à saúde; PCT: Programa de Controle da Tuberculose; TB: tuberculose; TRM/TB: teste rápido molecular.

* Melhora com Estratificação por Grau de Risco Clínico e de Abandono do Tratamento da Tuberculose. 
fissionais do NASF desenvolvem suas funções em dois a três centros de saúde, e a recomendação de envolvê-los no atendimento ao doente de TB foi bastante disseminada no município, após a ERTB 3.

No eixo Estrutura - Organização do Serviço, com relação ao horário de atendimento, quando esse é estendido para todo o Centro de Saúde, há uma melhora do vínculo do paciente com o serviço e melhora do acesso, o que não acontecia antes da ERTB, quando o horário era restrito ao atendimento da EqSF. Alguns autores descrevem que operacionalizar as atividades do Centro de Saúde com as necessidades da população e disponibilizar atendimento contínuo a pacientes com TB melhoram adesão 17,21,22, porém não foi a proposta deste estudo.

Em relação a "Articulação de todos os serviços de APS com os outros níveis de atenção", foi observado que os pacientes foram encaminhados para outras especialidades, setores ou serviços, o que favoreceu a comunicação entre esses profissionais, influenciando o desfecho favorável na segunda entrevista. Portanto, é indicado que as articulações devam ser sistematizadas e registradas para uma melhor efetividade do programa de TB 19,23,24,25.

Os resultados de exames bacteriológicos são inseridos eletronicamente no Sistema de Saúde em Rede (SISREDE) da Prefeitura de Belo Horizonte em até sete dias. Essa é uma normatização antiga da gestão do Programa Municipal de Controle da Tuberculose (PMCT) com a equipe do Apoio Diagnóstico 10. Os resultados positivos são enviados aos gerentes dos centros de saúde em menos de três dias, com o objetivo de iniciar o tratamento o mais precocemente possível. Com a ERTB esses resultados passaram a ser entregues imediatamente aos profissionais, resultando no início de tratamento mais precoce, minimizando o tempo de espera e consequentemente a transmissão da doença. Estudos realizados para avaliação das ações e estratégias para o controle da TB descrevem a importância dos serviços informatizados para a qualidade e continuidade no atendimento à pessoa com TB 26,27.

Nos eixos Estrutura - Recursos Físicos e Organização do Serviço, o desempenho foi satisfatório em quase todas as variáveis, demonstrando a boa estruturação dos serviços de APS em relação à TB, diferente do relatado em outros estudos 18,19. É importante salientar que a Prefeitura de Belo Horizonte tem investido ao longo dos anos nos sistemas de informação, disponibilização de insumos e medicamentos.

Quando analisado o eixo Processo - Atenção Proporcionada, também houve desempenho satisfatório para a maioria das variáveis, pois o PMCT já é estruturado com protocolos padronizados e disponíveis. Ressaltando que a monitorização dos processos de trabalho pode influenciar o sucesso das ações de controle da TB.

Em relação ao eixo Estrutura - Recursos Humanos, fragilidades foram encontradas no que se refere ao envolvimento de outros profissionais, principalmente do NASF (farmacêutico, assistente social e psicólogo) e capacitação em TB. Assim como em outros estudos 18,19,20, a resistência e rotatividade dos profissionais ao atendimento à pessoa com $\mathrm{TB}$, sobrecarga de serviço e falta de recursos humanos influenciaram no desempenho dos serviços. Estudos realizados em várias regiões do país reforçam que a descontinuidade das ofertas de capacitação, ausência de políticas de educação permanente, rotatividade dos profissionais e descontinuidade político-partidária podem configurar um problema na qualificação dos profissionais para atender as pessoas com TB 18,27,28. A TB é uma doença intimamente ligada a questões sociais 1 , sendo necessário suporte social o que indica que o assistente social deve estar envolvido no acompanhamento dos casos 29 . Portanto, os resultados obtidos neste estudo ressaltam a importância do aumento do número de profissionais nas equipes do NASF, principalmente assistente social e psicólogo, além de incluir o tema "tuberculose" na Política de Educação Permanente municipal. $\mathrm{O}$ trabalho em equipe e intersetorial é fundamental para a execução das ações de controle da TB, assim como para o êxito do tratamento e acompanhamento dos casos 29 .

Na Secretaria Municipal de Saúde não existem protocolos de dispensação de cestas básicas e vale-transporte, variáveis do eixo Estrutura - Recursos Físicos. Porém, existem outros protocolos que contemplam a necessidade do doente, tais como dispensação de cestas básicas pela Secretaria de Assistência Social, Segurança Alimentar e Cidadania após relatório social e disponibilização de transporte sanitário, mas não específico aos pacientes com TB 30. Existem ainda outros benefícios e/ ou transferências de renda que o assistente social avalia, orientando e auxiliando qualquer paciente a receber 31. Alguns estudos mais recentes realizados em países de baixa renda e alta carga, incluindo o Brasil, relatam que pacientes beneficiários de programa de transferência de renda têm uma chance de sucesso no tratamento maior do que aqueles que não recebem, visto que a TB está diretamente 
relacionada às condições sociais 31,32,33. Logo, é recomendável que todo paciente com diagnóstico de TB seja encaminhado para uma avaliação do serviço social.

Ao avaliarmos o eixo Processo - Atenção Proporcionada, os serviços de APS de Belo Horizonte precisam avançar nas variáveis "Oferta de tratamento diretamente observado no serviço de saúde, Oferta do tratamento diretamente observado no trabalho do doente de TB, Realização mínima de baciloscopia mensal de escarro para controle do tratamento e Existência de contrarreferência das informações".

Neste estudo, como em outros trabalhos 18,19, foi identificado que os profissionais ofertam o tratamento diretamente observado, com maior frequência, apenas no domicílio. No entanto, o tratamento diretamente observado deve ser ofertado em qualquer local acessível ao paciente, como descrito no Manual de Recomendações para o Controle da Tuberculose no Brasil 3. Cabe às EqSF uma organização maior no sentido de ampliação da oferta do tratamento diretamente observado, seja nos próprios centros de saúde ou em outro local indicado pelo paciente. É importante que essa ação ocorra em comum acordo entre paciente e equipe de saúde de forma a oportunizar o cuidado, sob a coordenação da APS. Outra recomendação importante para o acompanhamento dos casos com TB pulmonar com confirmação laboratorial é a realização de uma baciloscopia mensal de controle, porém os pacientes podem não apresentar escarro espontâneo após o primeiro mês de tratamento 3 .

Outra importante observação deste estudo é o fato de que o sistema de contrarreferência das informações por escrito não funciona adequadamente. Com o avanço das tecnologias de informação, urge a implantação de um sistema integrado e informatizado para todos os níveis de atenção, independentemente do nível de gestão. Com isso, o acesso aos dados será facilitado para melhor acompanhamento dos pacientes. Isso também acontece em outras condições de saúde (diferentes da TB), em que o sistema de informação de referência e contrarreferência é um grande problema. Tais achados vão ao encontro de resultados de outros estudos envolvendo os serviços de APS 18,26.

Em relação à disponibilidade de frasco de coleta para exame de escarro, no momento da segunda entrevista, ocorreu o desabastecimento temporário desse insumo no município, justificado pela quebra de contrato do fornecedor, fato que expõe fragilidades que perpassam o controle da TB em Belo Horizonte.

No que tange aos recursos físicos, organização do serviço e atenção proporcionada, torna-se evidente o quão a APS de Belo Horizonte é robusta e organizada 10,30, mas ainda necessita de investimento na Política de Educação Permanente voltada para o agravo TB, além de estratégias para o envolvimento de toda a equipe dos centros de saúde para as ações de controle da TB.

Este estudo apresentou algumas limitações, como a impossibilidade de incluir os profissionais que não responderam à primeira e à segunda entrevistas (133/588 - 23\%) em virtude da mudança de EqSF, o que pode ter interferido nos resultados. Outra limitação foi a avaliação dos usuários, que poderia contribuir na melhoria dos eixos Estrutura e Processo.

Concluímos que foi possível identificar o desempenho satisfatório na maioria das variáveis avaliadas nos eixos Estrutura e Processo, dos serviços de APS em Belo Horizonte em relação às ações de controle da TB, por meio de um questionário padronizado. Trata-se de uma ferramenta eficaz para dar respostas sobre as ações planejadas e executadas, além de identificar as falhas e qualificar o atendimento. 


\section{Colaboradores}

J. V. C. Rabelo coletou os dados, construiu o banco de dados, acompanhou as análises, redigiu e participou de todas as etapas de revisão do artigo. P. D. Navarro participou da concepção e desenvolvimento do estudo e revisão crítica do artigo. W. S. Carvalho e S. S. Miranda coordenaram o estudo, desenharam o método, coordenaram as atividades, acompanharam as análises e participaram da redação e aprovação do artigo. I. N. Almeida participou da revisão bibliográfica, análise crítica do artigo e revisão da versão final. C. S. F. Oliveira e J. P. A. Haddad realizaram as análises estatísticas e participaram da redação do artigo.

\section{Informações adicionais}

ORCID: Juliana Veiga Costa Rabelo (0000-00017599-5565); Pedro Daibert de Navarro (0000-00033267-4985); Wânia da Silva Carvalho (0000-00022575-6352); Isabela Neves de Almeida (0000-00016152-7648); Camila Stefânie Fonseca Oliveira (0000-0002-5557-7267); João Paulo Amaral Haddad (0000-0003-2823-6288); Silvana Spindola de Miranda (0000-0001-7245-4472).

\section{Agradecimentos}

Agradecemos ao Professor Antônio Ruffino Netto pela disponibilidade em nos apoiar e avaliar o conteúdo do artigo finalizado. À Rede Brasileira de Tuberculose - REDE TB. Ao Programa de Controle da Tuberculose e Hanseníase da Prefeitura Municipal de Belo Horizonte. Ao Programa de Pós-graduação em Ciências da Saúde: Infectologia e Medicina Tropical da Faculdade de Medicina da Universidade Federal de Minas Gerais (FAPEMIG - APQ 03266-13/APQ 00094-12. CNPq 446796/2014 e 310174/2017-7), Ministério da Saúde.

\section{Referências}

1. World Health Organization. Global tuberculosis report 2019. Geneva: World Health Organization; 2019.

2. WHO declares tuberculosis a global emergency. Soz Präventivmed 1993; 38:251-2.

3. Departamento de Vigilância das Doenças Transmissíveis, Secretaria de Vigilância em Saúde, Ministério da Saúde. Manual de recomendações para o controle da tuberculose no Brasil. Brasília: Ministério da Saúde; 2019.

4. Organización Panamericana de la Salud. Plan regional de tuberculosis 2006-2015. Washington DC: Organización Panamericana de la Salud; 2006.

5. World Health Organization. Guidelines on tuberculosis infection prevention and control 2019. Geneva: World Health Organization; 2019.

6. Mendes EV. As redes de atenção à saúde. Brasília: Organização Pan-Americana da Saúde; 2011.

7. Andrade RLP, Scatolin BE, Wysocki AD, Beraldo AA, Monroe AA, Scatena LM, et al. Diagnóstico da tuberculose: atenção básica ou pronto atendimento? Rev Saúde Pública 2013; 47:1149-58.

8. Organização Mundial da Saúde. Cuidados inovadores para condições crônicas: componentes estruturais de ação: relatório mundial. Brasília: Organização Mundial da Saúde; 2002.

9. Mendes EV. As redes de atenção à saúde. Ciênc Saúde Colet 2010; 15:2297-305.

10. Secretaria Municipal de Saúde, Prefeitura Municipal de Belo Horizonte. Atenção Primária. https://prefeitura.pbh.gov.br/saude/informa coes/atencao-a-saude/atencao-primaria (acessado em 04/Mai/2020).

11. Departamento de Doenças de Condições Crônicas e Infecções Sexualmente Transmissíveis, Secretaria de Vigilância em Saúde, Ministério da Saúde. Boletim epidemiológico especial. https://antigo.saude.gov.br/images/pdf/2020/ marco/24/Boletim-tuberculose-2020-mar cas--1-.pdf (acessado em 16/Fev/2020)

12. Silva CHL. Proposta de implantação de um novo modelo de atenção ao paciente com tuberculose, estruturado em redes, como estratégia para fortalecer a estratégia dots - "directly observed therapy short-couse". Lisboa: Instituto Universitário de Lisboa; 2010.

13. Ministério da Saúde. Portaria no 3.110, de 17 de dezembro 2013. Autoriza o repasse financeiro do Fundo Nacional de Saúde aos Fundos de Saúde dos Municípios com alta carga da doença para implantação/implementação de ações contingenciais de vigilância, prevenção e controle da tuberculose. Diário Oficial da União 2013; 18 dez.

14. Prefeitura Municipal de Belo Horizonte. Índice de Vulnerabilidade da Saúde (IVS-BH). https://prefeitura.pbh.gov.br/estatisticas-e-in dicadores/indice-de-vulnerabilidade-da-sau de (acessado em 16/Fev/2020). 
15. Villa TCS, Ruffino-Netto A. Questionário para avaliação de desempenho de serviços de atenção básica no controle da tuberculose no Brasil. J Bras Pneumol 2009; 35:610-12.

16. Samico I, Hartz ZMA, Felisberto EF. Atenção à saúde da criança: grau de implantação e da satisfação de profissionais e usuários em dois municípios do estado de Pernambuco, Brasil. Rev Bras Saúde Matern Infant 2005; 5:229-40.

17. Felisberto E, Carvalho EF, Maggi RS, Samico I. Avaliação do processo de implantação da estratégia da Atenção Integrada às Doenças Prevalentes da Infância no Programa Saúde da Família, no Estado de Pernambuco, Brasil. Cad Saúde Pública 2002; 11:1737-45.

18. Silva DM. Tratamento da tuberculose na Atenção Básica: avaliação da estrutura e processo dos serviços de saúde [Dissertação de Mestrado]. João Pessoa: Programa de Pós Graduação em Enfermagem, Centro de Ciências da Saúde, Universidade Federal da Paraíba; 2013.

19. Wysocki AD, Ponce MAS, Brunello MEF, Beraldo AA, Vendramini SHF, Scatena LM, et al. Atenção Primária à Saúde e tuberculose: avaliação dos serviços. Rev Bras Epidemiol 2017; 20:161-75.

20. Ponce MA, Wysocki AD, Scatolin BS, Andrade RLP, Arakawa T, Ruffino-Netto A, et al. Diagnóstico da tuberculose: desempenho do primeiro serviço de saúde procurado em São José do Rio Preto, São Paulo, Brasil. Cad Saúde Pública 2013; 29:945-54.

21. Scatena LM, Wysocki AD, Beraldo AA, Magnabosco GT, Brunello ME, Ruffino-Netto A, et al. Validação e confiabilidade: instrumento para avaliação de serviços que tratam tuberculose. Rev Saúde Pública 2015; 49:7.

22. Marcolino ABL, Nogueira JA, Ruffino-Netto A, Moraes RM, Sá LD, Villa TCS, et al. Avaliação do acesso à ações de controle da tuberculose no contexto das equipes de saúde da família de Bayeux-PB. Rev Bras Epidemiol 2009; 12:144-57.

23. Hartz ZM. Avaliação em saúde. Rio de Janeiro: Editora Fiocruz; 1997.

24. Hartz ZM. Princípios e padrões em metavaliação: diretrizes para os programas de saúde. Ciênc Saúde Colet 2006; 11:733-38.
25. Contandriopoulos AP. Avaliando a institucionalização da avaliação. Ciênc Saúde Colet 2006; 10:705-11.

26. Assis EG, Beraldo AA, Monroe AA, Scatena LM, Cardozo-Gonzales RI, Palha PF, et al. A coordenação da assistência no controle da tuberculose. Rev Esc Enferm USP 2012; 45:1118.

27. Monroe AA, Cardozo Gonzales RI, Palha PF, Sassaki CM, Ruffino Netto A, Vendramini SHF, et al. Envolvimento de equipes da Atenção Básica à Saúde no controle da tuberculose. Rev Esc Enferm USP 2008; 42:262-67.

28. Façanha MC, Melo MA, Vasconcelos FF, Sousa JRP, Pinheiro AS, Porto IA, et al. Treinamento da equipe de saúde e busca ativa na comunidade: estratégias para a detecção de casos de TB. J Bras Pneumol 2009; 35:449-54.

29. Mazzei AMA, Monroe AA, Sassaki CM, Gonzales RIC, Villa TCS. Suporte social para portador de tuberculose no serviço de saúde e na comunidade. Bol Pneumol Sanit 2003; 11:416.

30. Secretaria Municipal de Assistência Social, Prefeitura Municipal de Belo Horizonte. Segurança alimentar e cidadania. https://pre feitura.pbh.gov.br/smasac (acessado em 05/ Mai/2020).

31. Brasil. Lei no 8.742, de 7 de dezembro de 1993. Dispõe sobre a organização da Assistência Social e dá outras providências. Diário Oficial da União 1993; 10 dez.

32. Andrade KVF, Nery JS, Souza RA, Pereira SM. Effects of social protection on tuberculosis treatment outcomes in low or middle-income and in high-burden countries: systematic review and meta analysis. Cad Saúde Pública 2018; 34:e00153116.

33. Oliosi JGN, Santos BR, Locatelli RL, Sales CMM, Silva Filho WG, Silva KC, et al. Effect of the Bolsa Familia Programme on the outcome of tuberculosis treatment: a prospective cohort study. Lancet Glob Health 2019; 7:219-26. 


\begin{abstract}
The World Health Organization highlights the importance of health services organization and performance in tuberculosis (TB) control activities. This study aimed to assess the performance of primary healthcare services in Belo Horizonte, Minas Gerais State, Brazil, in TB control activities in the dimensions Structure and Process, before and after the use of a validated instrument called Stratification by Degree of Clinical Risk and Tuberculosis Treatment Dropout (ERTB). This was a descriptive and prospective study with two interviews (455 professionals), the second of which after the ERTB. Performance classification was: $\leq 49.9 \%$, critical; 50-79.9\%, unsatisfactory, and $\geq 80 \%$, satisfactory. The comparative assessment used the McNemar statistical test with $p<$ 0.05 . After risk stratification, most of the variables in each dimension improved significantly. The authors conclude that it was possible to identify satisfactory performance in most of the variables assessed in the Structure and Process dimensions in primary healthcare services in Belo Horizonte in relation to $T B$ control activities, using a standardized questionnaire.
\end{abstract}

Tuberculosis; Health Services; Primary Health Care

\section{Resumen}

La Organización Mundial de la Salud destaca la importancia de la organización y del desempeño de los servicios de salud en acciones de control de la tuberculosis. El objetivo de este estudio fue evaluar el desempeño de los servicios de atención primaria en salud en Belo Horizonte, Minas Gerais, Brasil, en relación con las acciones de control de la tuberculosis respecto a los ejes Estructura y Proceso, antes y después de la utilización del instrumento validado, denominado Estratificación por Grado de Riesgo Clínico y de Abandono del Tratamiento de la Tuberculosis (ERTB). Se trata de un estudio descriptivo y prospectivo, donde se realizaron dos entrevistas (455 profesionales), siendo que la segunda se produjo tras la ERTB. La clasificación del desempeño siguió los valores: $\leq$ 49,9\%, críticos; entre 50 y 79,9\%, insatisfactorios; $y, \geq 80 \%$, satisfactorios. En la evaluación comparativa, se utilizó el test estadístico de McNemar, con $p<0,05$. Tras la estratificación de riesgo, la mayor parte de las variables de cada eje mejoró significativamente. Concluimos que fue posible identificar el desempeño satisfactorio en la mayoría de las variables evaluadas en los ejes Estructura y Proceso de los servicios de atención primaria en salud en Belo Horizonte, en relación con las acciones de control de la tuberculosis mediante un cuestionario estandarizado.

Tuberculosis; Servicios de Salud; Atención Primaria de Salud

Recebido 05/Mai/2020
Versão final reapresentada em 28/Jul/2020

Aprovado em 31/Jul/2020 